Ophthalmologe 2022 $\cdot 119: 730-732$ https://doi.org/10.1007/s00347-021-01408-2 Eingegangen: 12. Februar 2021

Überarbeitet: 11. April 2021

Angenommen: 26. April 2021

Online publiziert: 12. Mai 2021

() Der/die Autor(en) 2021
Peter Werkl ${ }^{1}$ Nora Woltsche ${ }^{1}$ Barbara Silbernagel ${ }^{2}$ Gernot Schließleder ${ }^{1}$. Wolfgang List ${ }^{1} \cdot$ Gernot Steinwender ${ }^{1} \cdot$ Ingrid Boldin $^{1} \cdot$ Jutta Horwath-Winter ${ }^{1}$ ${ }^{1}$ Universitätsaugenklinik, Medizinische Universität Graz, Graz, Österreich

${ }^{2}$ Univ.-Klinik für Innere Medizin, Klinische Abteilung für Hämatologie, Medizinische Universität Graz, Graz, Österreich

\title{
Neue Therapien stellen uns vor neue Herausforderungen
}

\section{Okuläre Nebenwirkungen durch Belantamab Mafodotin}

\section{Anamnese}

Im Mai 2020 stellte sich ein 76-jähriger männlicher Patient mit leichtem Fremdkörpergefühl an beiden Augen in unserer Ambulanz vor. Er gab keine Sehverschlechterung an. In der ophthalmologischen Anamnese bestanden keinerlei Auffälligkeiten in der Vorgeschichte. Im Rahmen der Erhebung der Allgemeinanamnese stellte sich jedoch heraus, dass der Patient im Dezember 2018 die Diagnose eines multiplen Myeloms von Typ IgA/Lambda erhalten hatte. Nach verschiedenen Vortherapien - darunter VRD (Bortezomib/Lenalidomid/ Dexamethason), autologer Stammzelltransplantation sowie Daratumumab war Mitte April 2020 Belantamab Mafodotin eingeleitet worden. Es wurden 3 Zyklen von Belantamab Mafodotin verabreicht, jedoch musste die Behandlung Ende Mai 2020 aufgrund des Rezidivs eines Urothelkarzinoms abgebrochen werden.

\section{Befunde}

In der Spaltlampenuntersuchung zeigte sich bei Erstvorstellung lediglich eine mäßige korneale Stippung inferior beidseits, woraufhin eine konservierungsmittelfreie Benetzungstherapie eingeleitet wurde. Mitte Juni erfolgte dann jedoch eine Wiedervorstellung, bei der vom Patienten eine Sehverschlechterung beidseits angegeben wurde. Der korrigierte Visus betrug nun 0,63/0,8.
An diesem Tag zeigten sich erstmals intraepitheliale korneale Zysten in der mittleren Peripherie an beiden Augen zusätzlich zu einer wirbelförmigen Stippung (-Abb. 1 und 2). Die basalen intraepithelialen Zysten konnten auch in der konfokalen Mikroskopie, welche im Rahmen der erweiterten Diagnostik durchgeführt wurde, visualisiert werden (-Abb. 3a, b). Der Schirmer-Test ohne Lokalanästhesie betrug $7 / 14 \mathrm{~mm}$, die T-BUT (Tränenfilmaufreißzeit) $3 \mathrm{~s}$ beidseits.

\section{Diagnose}

Epitheliale Mikrozysten der Kornea in Assoziation mit dem AntikörperWirkstoff-Konjugat Belantamab Mafodotin (BLENREP, GlaxoSmithKline plc, Brentford, UK).

Bei den beschriebenen bilateralen intraepithelialen Mikrozysten der Hornhaut sollte differenzialdiagnostisch auch an eine Meesmann-Dystrophie gedacht werden. Hierbei handelt es sich um eine autosomal-dominante und nichtprogressive Hornhauterkrankung. Der Spaltlampenbefund imponiert ähnlich, und in der konfokalen Mikroskopie zeigen sich üblicherweise hyporeflektive rundliche Areale im basalen kornealen Epithel mit einer Größe zwischen 48 und $145 \mu \mathrm{m}$ mit reflektiven Punkten [3]. Der zugrunde liegende genetische Defekt liegt in den Genen KRT3 sowie KRT12 [1]. Die genetische Testung des hier beschriebenen Patienten auf eine Mutation in KRT3 und KRT12 zeigte einen unauffälligen Befund.

\section{Therapie und Verlauf}

Zusätzlich zur konservierungsmittelfreien Benetzungstherapie wurden nun Dexamethason-Augentropfen 3-mal täglich eingeleitet, welche die Progression der Zysten Richtung Zentrum der Kornea und die weitere Sehverschlechterung auf 0,63 cc beidseits bis Anfang Juli jedoch nicht verhindern konnten. Auch hyperosmolare Augentropfen führten bis Mitte Juli zu keiner Veränderung der intraepithelialen Zysten. Im weiteren Verlauf wurden die Dexamethason-Augentropfen ebenso wie die hyperosmolaren $\mathrm{Au}$ gentropfen aufgrund einer ausbleibenden Befundverbesserung abgesetzt. Da sich auch nach Absetzen ebendieser Therapie keine Verschlechterung beim letzten Kontrolltermin Ende Juli zeigte, wurde die alleinige befeuchtende Lokaltherapie beibehalten. Der Patient verstarb leider im Herbst 2020 an seiner Grunderkrankung.

\section{Diskussion}

Bei dem Medikament Belantamab Mafodotin handelt es sich um ein Immunkonjugat zwischen Anti-BCMA („B-cell maturation antigen" [BCMA]) und Monomethyl-Auristatin-F (MMAF, eine Substanz, welche die Zellteilung durch Inhibition der Polymerisation der Mikrotubuli hemmen soll), welches in Phase I 


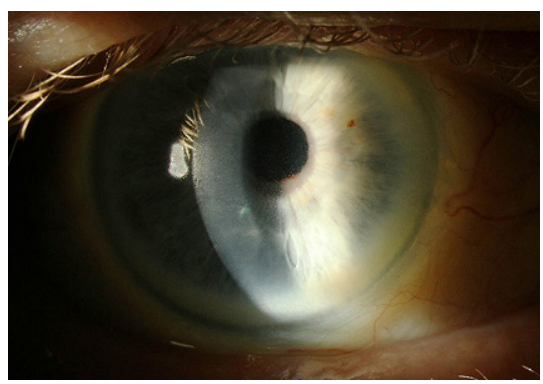

Abb. 1 ॥ Spaltlampenfoto Hornhaut RA - intraepitheliale Mikrozysten

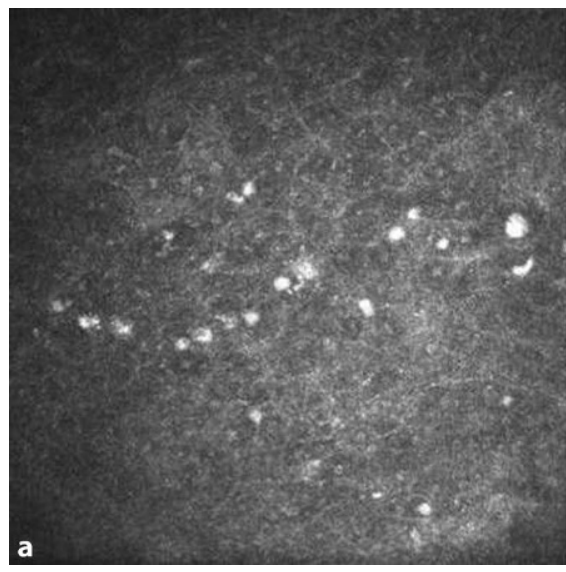

Abb. $3 \Delta$ a, b Konfokale Mikroskopie (Rostock Cornea Modul, Heidelberg Engineering) Hornhaut RA hyperreflektive Zysten im basalen Epithel

und II bis dato überzeugende Therapieerfolge bei intensiv vorbehandelten, therapierefraktären PatientInnen mit multiplem Myelom zeigen konnte.

Einzelne Studien berichten über die Entstehung von bilateralen epithelialen Mikrozysten als häufige Nebenwirkung meist früh nach Therapiebeginn mit Belantamab Mafodotin in bis zu $72 \%$ der PatientInnen, häufig mit Symptomen wie Verschwommensehen und/oder Augentrockenheit einhergehend $[2,5]$. Der genaue Pathomechanismus ist bis dato ungeklärt, jedoch wird ein unspezifischer, BCMA-unabhängiger Uptake der Konjugate in aktiv in Teilung befindliche basale epitheliale Stammzellen der Kornea als Ursache vermutet [4].

Prävention: Die DREAMM-2-Studie konnte keinen Vorteil einer prophylaktischen Behandlung mit kortisonhaltigen Augentropfen gegenüber einer alleinigen konservierungsmittelfreien Benetzungstherapie zeigen $[4,5]$. Alle PatientInnen sollten zum Zeitpunkt der Therapieinitiierung beginnen, mindestens 4-mal
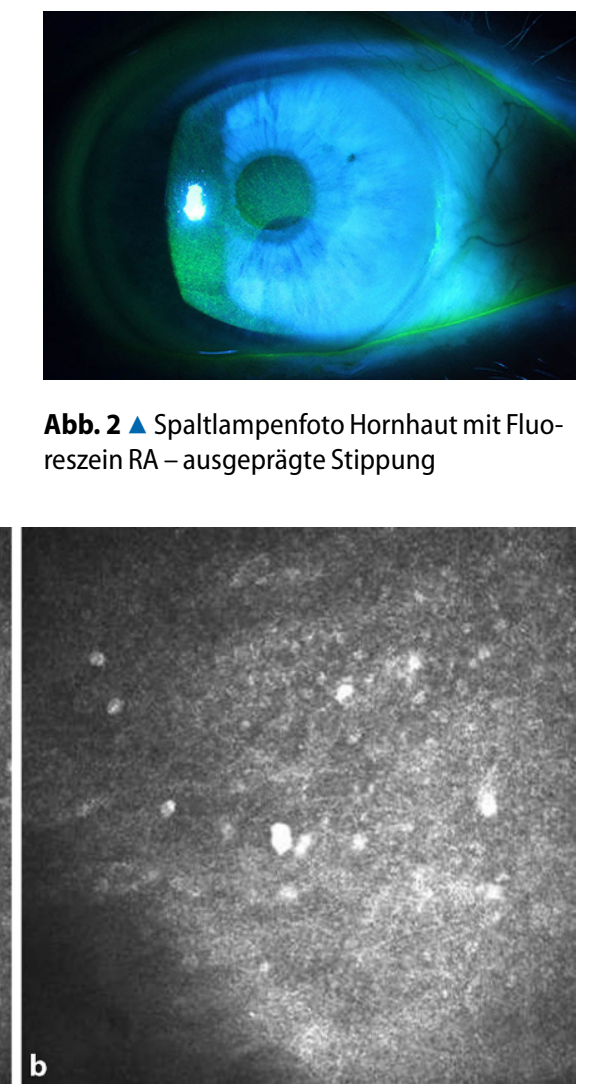

Abb. $2 \Delta$ Spaltlampenfoto Hornhaut mit Fluoreszein RA - ausgeprägte Stippung

täglich konservierungsmittelfreie Benetzungstropfen $\mathrm{zu}$ applizieren. Dies sollte über die gesamte Therapiedauer beibehalten werden. Während der Behandlung sollten keine Kontaktlinsen getragen werden. Um korneale Nebenwirkungen schnell erkennen zu können, sollten PatientInnen, welche Belantamab Mafodotin erhalten, vor Therapiebeginn, vor den 3 nachfolgenden Behandlungszyklen sowie beim Auftreten okulärer Symptome ophthalmologisch untersucht werden (Sehschärfe sowie Spaltlampenuntersuchung inklusive Fluoreszeinfärbung der Augenoberfläche). Besonders PatientInnen mit trockenem Auge vor Therapiebeginn mit Belantamab Mafodotin haben ein höheres Risiko für korneale Nebenwirkungen [2].

Einige Wochen bis Monate nach Therapiebeendigung von anderen mit MMAF assoziierten Antikörperkonjugaten bilden sich im Verlauf aufgetretene epitheliale Mikrozysten komplett zurück. Dieser Zeitraum ist konsistent mit der Kinetik der Repopulation des kornealen
Epithels durch Stammzellen. Es wurde ein genauer Handlungsalgorithmus je nach vorliegendem Grad der okulären Nebenwirkungen etabliert: bei Grad 1 (milde Stippung, Visusverschlechterung um 1 Zeile) Beibehalten der Dosis; bei Grad 2 (moderate Stippung - lokalisierte epitheliale Mikrozysten, subepithelialer Haze, neue periphere stromale Trübung; Visusverschlechterung um 2 bis 3 Zeilen) Verschieben des nächsten Zyklus bis zur Verbesserung des Befunds auf Grad 1, dann Weiterbehandlung mit derselben Dosis wie zuvor; bei Grad 3 (schwere Stippung - diffuse zentrale epitheliale Mikrozysten, zentraler subepithelialer Haze, neue zentrale stromale Trübung; Visusverschlechterung um mehr als 3 Zeilen) ebenfalls Verschiebung des nächsten Zyklus bis zur Verbesserung des Befunds auf Grad 1, danach reduzierte Dosis. Grad 4 (Ulcus corneae) bedingt je nach Nutzen-Risiko-Abwägung in den meisten Fällen einen Therapieabbruch [2].

Bei diesem Fall handelt es sich um keine alltägliche Diagnose, jedoch zeigt er die Wichtigkeit einer genauen Anamnese und der Kenntnis laufend neuer in anderen Fachdisziplinen auf den Markt kommender Wirkstoffe auch für uns AugenärztInnen auf. Auch Antikörperkonjugierte Substanzen stehen aktuell im Fokus der Wirkstoffentwicklung und können nicht selten zu okulären $\mathrm{Ne}$ benwirkungen führen, wie es auch bei unserem Patienten unter Belantamab Mafodotin der Fall war. Daher sollte bereits durch den/die betreuenden HämatologIn vor Therapiebeginn über die möglichen kornealen Nebenwirkungen und deren Symptome aufgeklärt werden. Auch sollten regelmäßige ophthalmologische Kontrollen während der Therapie erfolgen. Diesbezüglich wurde vom Hersteller im Rahmen der Zulassung eine Informationsbroschüre über die möglichen okulären Nebenwirkungen zur Verfügung gestellt. Diese ist unter anderem auf der Homepage des Paul-Ehrlich-Institutes $\mathrm{zu}$ finden und bietet einen Leitfaden für PatientInnen und betreuende ÄrztInnen. 


\section{Termine}

\section{Korrespondenzadresse}

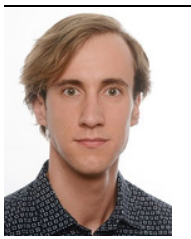

Dr. med. univ. Peter Werkl

Universitätsaugenklinik,

Medizinische Universität Graz

Graz, Österreich

peter.werkl@medunigraz.at

Funding. Open access funding provided by Medical University of Graz.

\section{Einhaltung ethischer Richtlinien}

Interessenkonflikt. P. Werkl, N. Woltsche, B. Silbernagel, G. Schließleder, W. List, G. Steinwender, I. Boldin und J. Horwath-Winter geben an, dass kein Interessenkonflikt besteht.

Für diesen Beitrag wurden von den Autoren keine Studien an Menschen oder Tieren durchgeführt. Für die aufgeführten Studien gelten die jeweils dort angegebenen ethischen Richtlinien. Für Bildmaterial oder anderweitige Angaben innerhalb des Manuskripts, über die Patienten zu identifizieren sind, liegt von ihnen und/oder ihren gesetzlichen Vertretern eine schriftliche Einwilligung vor.

Open Access. Dieser Artikel wird unter der Creative Commons Namensnennung 4.0 International Lizenz veröffentlicht, welche die Nutzung, Vervielfältigung, Bearbeitung, Verbreitung und Wiedergabe in jeglichem Medium und Format erlaubt, sofern Sie den/die ursprünglichen Autor(en) und die Quelle ordnungsgemäß nennen, einen Link zur Creative Commons Lizenz beifügen und angeben, ob Änderungen vorgenommen wurden.

Die in diesem Artikel enthaltenen Bilder und sonstiges Drittmaterial unterliegen ebenfalls der genannten Creative Commons Lizenz, sofern sich aus der Abbildungslegende nichts anderes ergibt. Sofern das betreffende Material nicht unter der genannten Creative Commons Lizenz steht und die betreffende Handlung nicht nach gesetzlichen Vorschriften erlaubt ist, ist für die oben aufgeführten Weiterverwendungen des Materials die Einwilligung des jeweiligen Rechteinhabers einzuholen.

Weitere Details zur Lizenz entnehmen Sie bitte der Lizenzinformation auf http://creativecommons.org/ licenses/by/4.0/deed.de.

\section{Literatur}

1. Chen JL, Lin BR, Gee KM et al (2015) Identification of presumed pathogenic KRT3 and KRT12 gene mutations associated with Meesmann corneal dystrophy. Mol Vis 21:1378-1386

2. Farooq AV, Degli Esposti S, Popat R et al (2020) Corneal epithelial findings in patients with multiple myeloma treated with antibody-drug conjugate belantamab mafodotin in the pivotal, randomized, DREAMM-2 study. Ophthalmol Ther 9:889-911. https://doi.org/10.1007/s40123-02000280-8
3. Patel DV, Grupcheva CN, McGhee CNJ (2005) Imaging the microstructural abnormalities of Meesmann corneal dystrophy by in vivo confocal microscopy. Cornea 24:669-673. https://doi.org/ 10.1097/01.ico.0000154389.51125.70

4. Popat R, Warcel D, O'Nions J et al (2020) Characterization of response and corneal events with extended follow-up after belantamab mafodotin (GSK2857916) monotherapy for patients with relapsed multiple myeloma: a case series from the first-time-in-human clinical trial. Haematologica 105:e261-e263. https://doi.org/10.3324/ haematol.2019.235937

5. Lonial S, Lee HC, Badros A et al (2020) Belantamab mafodotin for relapsed or refractory multiple myeloma (DREAMM-2): a two-arm, randomised, open-label, phase 2 study. Lancet Oncol 21(2):207-221. https://www.thelancet.com/ journals/lanonc/article/PIIS1470-2045(19)30788$0 /$ fulltext

\section{Terminankündigung}

2022

DOG 2022

Berlin, 29.09. - 02.10.2022

https://dog-kongress.de/

2023

AAD 2023

Düsseldorf, 15.03. - 18.03.2023

https://aad-kongress.de/

DOG 2023

Berlin, 28.09. - 01.10.2023

https://dog-kongress.de/

2024

AAD 2024

Düsseldorf, 12.03. - 16.03.2024

https://aad-kongress.de/

DOG 2024

Berlin, 10.10. - 13.10.2024

https://dog-kongress.de/
Alle Angaben ohne Gewähr - Änderungen vorbehalten. 